\section{High-throughput generation of sequence indexes from T-DNA mutagenized Arabidopsis thaliana lines}

\author{
Nicolai Strizhov, Yong Li, Mario G. Rosso, Prisca Viehoever, Koen A. Dekker, \\ and Bernd Weisshaar \\ Max Planck Institute for Plant Breeding Research, Cologne, Germany \\ BioTechniques 35:1164-1168 (December 2003)
}

A pipeline has been created for the characterization of Arabidopsis thaliana mutants by generating flanking sequence tags (FSTs) and optimized for economic, high-throughput production. The GABI-Kat collection of T-DNA mutagenized A. thaliana plants was used as a source of independent transgenic lines. The pipeline included robotized extraction of genomic DNA in a 96-well format, an adapter-ligation PCR method for amplification of plant sequences adjacent to T-DNA borders, automated purification and sequencing of PCR products, and computational trimming of the resulting sequence files. Data quality was significantly improved by (i) restriction digestion of the adaptor-ligation products to reduce trivial sequences caused by co-amplification of fragments derived from the free plasmid, and (ii) the design of the adaptor primers for the second amplification step to enhance selective generation of single PCR fragments, even from lines with multiple T-DNA insertions. Gel-purification was avoided by including these steps, the number of amplification reactions per line was reduced from four to three, and the percentage of lines that yielded at least one FST was increased from $66 \%$ to $86 \%$. More than 58,000 FSTs have been submitted to GenBank ${ }^{\circledR}$ and are available at http://www.mpiz-koeln.mpg.de/GABI-Kat/.

\section{INTRODUCTION}

Random insertional mutagenesis, in which the foreign DNA not only disrupts a gene but also provides a tag for the characterization of the mutation, is used for the study of gene function relationships in a variety of organisms (1-4). In the model plant Arabidopsis thaliana, large collections of insertiontagged mutants have been established by Agrobacterium-based transformation (so called T-DNA mutants) (5-7). The publication of the complete $A$. thaliana genome sequence (8) stimulated the systematic identification of the T-DNA insertion sites by sequencing (9-11). However, there are several factors that have a negative effect on efforts to characterize such insertion-tagged populations by sequencing. T1 seeds (see Materials and Methods), and plants generated from these seeds are inhabited by the agrobacteria used in transformation. Thus, DNA extracted from T1 leaves can contain the T-DNA plasmid. Plasmid-derived sequences may amplify preferentially during recovery of DNA fragments from the
T-DNA insertion sites. Another reason for poor quality sequences are truncated, tandem, or multiple insertions that often occur in complex patterns, resulting in complicated amplification products (12). Separation of unwanted fragments by gel electrophoresis is tedious, while a low success rate in flanking sequence tag (FST) production is expensive.

We optimized the characterization process of $A$. thaliana T-DNA mutants by FST generation through the introduction of simple steps to deal with the above-mentioned problems in an economic way.

\section{MATERIALS AND METHODS}

\section{Plant Material and Growth Conditions}

A. thaliana ecotype Columbia (Col0 ) was used for plant transformation by Agrobacterium-mediated transfer with the flower-dip method (7). Plants containing the binary vector pAC161 (GenBank ${ }^{\circledR}$ accession no. AJ537514), conferring sulfadiazine resistance, were randomly selected from the GABI-Kat (http://www.mpiz-koeln.mpg.de/GABIKat// population. Plants were grown under natural greenhouse conditions with additional light to cover $16 \mathrm{~h}$.

\section{Isolation of Plant Genomic DNA}

Preparation of genomic DNA from 300-500 mg leaf material of T1 plants was adapted to 96-well polypropylene 2.2-mL deep well blocks (HJ-Bioanalytik, Moenchengladbach, Germany) and an automated Biomek FX 96channel pipettor (Beckman, Fullerton, CA, USA). We use the term T1 for the seeds and the plants that grow out of the embryos in these seeds, which are set by the infiltrated plants (T0 plants). T1 plants are hemizygous for the T-DNA insertion and contain the selectable marker. Blocks stored at $-80^{\circ} \mathrm{C}$ were cooled with liquid nitrogen, and leaf material was ground with two stainless steel ball bearings (outer diameter 3.5 $\mathrm{mm}$ ) per well by shaking for 30-45 s at $30 \mathrm{~Hz}$ in a Retsch 300 Matrix Mill homogenizer (Qiagen, Hilden, Germany).

Genomic DNA was prepared from ground leaf material according to a modified cetyltrimethylammonium bromide (CTAB)-DNA preparation protocol (13), replacing chloroform with dichloromethane $\left(\mathrm{CH}_{2} \mathrm{Cl}_{2}\right)$. Finally, 0.8-1.6 $\mu \mathrm{g}$ DNA was dissolved in $80 \mu \mathrm{L}$ TE $(10 \mathrm{mM}$ Tris-buffer, $0.1 \mathrm{mM}$ EDTA, $\mathrm{pH}$ 8.0) and stored at $-20^{\circ} \mathrm{C}$.

\section{Amplification of T-DNA Tagged Plant Sequences}

DNA amplifications were carried out in 96-well Thermo-Fast plates (ABgene, Epsom, Surray, UK) (14). About 100 ng of genomic DNA per line was digested with 4 to 5 U BfaI (New England Biolabs, Frankfurt, Germany) in $20 \mu \mathrm{L}$ total volume for approximately $5 \mathrm{~h}$ at $37^{\circ} \mathrm{C}$. The reaction mixture contained $5 \mu \mathrm{g}$ RNase A (Roche, Mannheim, Germany). An aliquot of 5-10 $\mu \mathrm{L}$ from the restriction reaction was used for ligation [20 $\mu \mathrm{L}$ total volume, approximately 3 Weiss $\mathrm{U}$ of T4 DNA ligase (New England Biolabs), for $11 \mathrm{~h}$ at $14^{\circ} \mathrm{C}$ ] with $12.5 \mathrm{pmol}$ of the adaptor T732/APL16OH. This asymmetrical adaptor was generated by the annealing of two oligonucleotides, T732 and APL16OH, at an equimolar 
Table 1. Sequences of the Oligonucleotides Used in this Study

\begin{tabular}{|c|c|c|}
\hline Name & Sequence & Purpose and Step Used \\
\hline T732 & 5'-GAGTAATACGACTCACTATAGGGAGGATCCCA-3' & Adaptor oligonucleotide, strand ligated to genomic DNA \\
\hline 8474 & 5'-ATAATAACGCTGCGGACATCTACATTTT-3' & LB primer, 2nd amplification \\
\hline 3087 & 5'-CCAAAGATGGACCCCCACCCAC-3' & RB primer, 1st amplification \\
\hline 2895 & 5'-CAGTCTCAGAAGACCAAAGGGC-3' & RB primer, 2nd amplification \\
\hline $\operatorname{adpA}$ & 5'-CTCACTATAGGGAGGATCCCATAGA-3' & Adaptor primer, 2nd amplification \\
\hline $\operatorname{adpC}$ & 5'-CTCACTATAGGGAGGATCCCATAGC-3' & Adaptor primer, 2nd amplification \\
\hline $\operatorname{adpG}$ & 5'-CTCACTATAGGGAGGATCCCATAGG-3' & Adaptor primer, 2nd amplification \\
\hline adpT & 5'-CTCACTATAGGGAGGATCCCATAGT-3' & Adaptor primer, 2nd amplification \\
\hline 8409 & 5'-ATATTGACCATCATACTCATTGC-3' & LB sequencing primer \\
\hline
\end{tabular}

concentration of $25 \mu \mathrm{M}$ (see Table 1 for all oligonucleotide sequences). Subsequently, the ligase was inactivated by incubation at $66^{\circ} \mathrm{C}$ for $17 \mathrm{~min}$. Five microliters of the ligation mixture was digested with either XhoI (for the left border, LB) or PaeI (for the right border, RB) for $5 \mathrm{~h}$ in $15 \mu \mathrm{L}$ at $37^{\circ} \mathrm{C}$. The first linear amplification was performed in $55 \mu \mathrm{L}$ with the whole XhoI or PaeI digest using primer 8603 (for LB) or primer 3087 (for RB) at a concentration of $200 \mathrm{nM}, 250 \mu \mathrm{M}$ of each dNTP, and 1.5 U Taq DNA polymerase (Invitrogen, Karlsruhe, Germany). Amplification was performed in a PTC-225 ${ }^{\circledR}$ Thermal Cycler (MJ Research, Waltham, MA, USA) using the following parameters: $94^{\circ} \mathrm{C}$ for $2 \mathrm{~min}, 30$ cycles of $94^{\circ} \mathrm{C}$ for 35 $\mathrm{s}, 64^{\circ} \mathrm{C}$ for $1 \mathrm{~min}$, and $73^{\circ} \mathrm{C}$ for $1.5 \mathrm{~min}$, and then elongation at $73^{\circ} \mathrm{C}$ for $3 \mathrm{~min}$. When postligation digestions were not performed, a $5-\mu \mathrm{L}$ aliquot of the ligation reaction was directly subjected to the first amplification. One microliter from the first amplification reaction was used as a template for the second PCR amplification in $50 \mu \mathrm{L}$ with nested T-DNA primers (8474 for LB and 2895 for RB) and adaptor primers adpA, adpC, adpG, adpT, or T726 at $200 \mathrm{nM}$ each. The PCR reagents and cycling parameters were identical to the first amplification step.

\section{Automated Sequencing and Computational Sequence Analysis}

The PCR products were purified over a Sephadex ${ }^{\text {TM }}$ G50 superfine col- umn (Amersham Biosciences, Freiburg, Germany). Dry Sephadex was filled into 96-well Multiscreen HV-plates (Millipore, Schwalbach, Germany), and $300 \mu \mathrm{L}$ water was added per well for swelling. After $3 \mathrm{~h}$, the plates were centrifuged at $910 \times g$ for $5 \mathrm{~min}$, washed with $150 \mu \mathrm{L}$ water, and again centri- 
Table 2. Effect of Various Pipeline Modifications on Final Flanking Sequence Tag Yield

\begin{tabular}{|c|c|c|c|c|}
\hline Methods & $\begin{array}{c}\text { Amplification } \\
\text { Reactions } \\
\text { per Line }\end{array}$ & $\begin{array}{c}\text { Sequencing } \\
\text { Reactions } \\
\text { per Line }\end{array}$ & $\begin{array}{l}\text { Genome Hits } \\
\text { per Line }\end{array}$ & $\begin{array}{l}\text { Lines with } \\
\text { Hits (\%) }\end{array}$ \\
\hline $\begin{array}{l}\text { II. Digestion (Xhol, LB; Pael, RB); second amplification with adaptor } \\
\text { primer T726 }\end{array}$ & 4 & 2 & 0.80 & 74 \\
\hline $\begin{array}{l}\text { IV. Digestion (Xhol, LB only); second amplification with adpA and } \\
\text { adpC or adpG and adpT }\end{array}$ & 3 & 2 & 1.05 & 86 \\
\hline $\begin{array}{l}\text { V. Digestion ( } P \text { ael, RB only); second amplification with adpA and } \\
\text { adpC or adpG and adpT }\end{array}$ & 3 & 2 & 0.26 & 25 \\
\hline $\begin{array}{l}\text { VI. Digestion (Xhol, LB; Pael, RB); second amplification with adpA } \\
\text { and adpC or adpG and adpT }\end{array}$ & 6 & 4 & 1.22 & 90 \\
\hline
\end{tabular}

fuged for $5 \mathrm{~min}$ at $910 \times g$. An aliquot of $16 \mu \mathrm{L}$ of the second amplification reaction was applied to the minicolumns and centrifuged, and $2.5 \mu \mathrm{L}$ of the eluate was used for cycle sequencing with the ABI PRISM ${ }^{\circledR}$ Big Dye Terminator Cycle Sequencing Ready Reaction kit v3.0 (Applied Biosystems, Weiterstadt, Germany) using primer 8409 for LB and primer 3144 for RB.
Unincorporated fluorescent nucleotides were removed by another centrifugation through a separate Sephadex minicolumn. The trace files generated by the ABI PRISM 96-capillary sequencer were fed into the computational data-processing pipeline (15). Information on the source plant of the PCR fragment analyzed was written into the respective trace file via the sample description

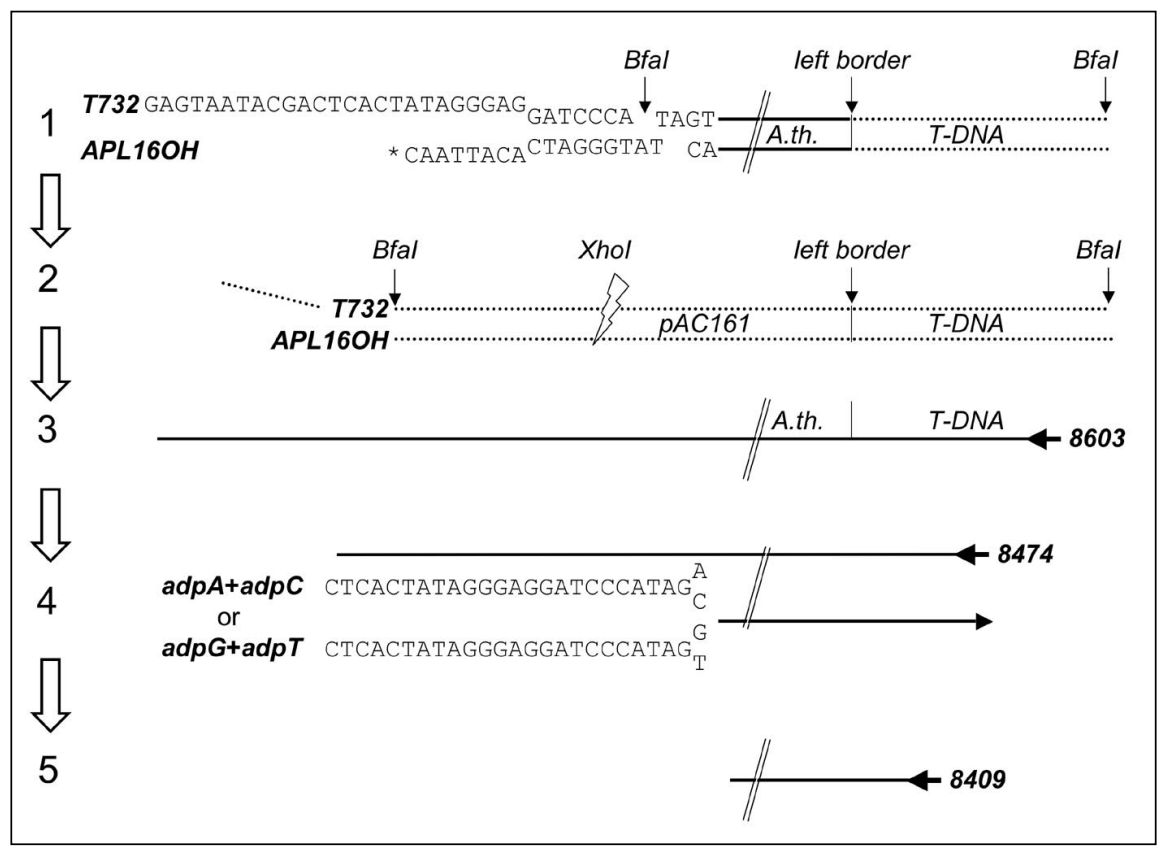

Figure 1. Overview of the final pipeline for the sequencing of genomic fragments flanking the known T-DNA insertion. The separate steps can be summarized as: (1) digestion of genomic DNA with $B f a \mathrm{I}$ and ligation of the asymmetrical adaptor T732/APL16OH; (2) removal of plasmid-derived fragments by digestion with XhoI; (3) linear amplification with T-DNA primer 8603; (4) PCR amplification with T-DNA primer 8474 and adaptor primers with A plus $\mathrm{C}$ or $\mathrm{G}$ plus $\mathrm{T}$; (5) cycle sequencing with T-DNA primer 8409. A. th., Arabidopsis thaliana. file of the sequencer. Each resulting sequence was assayed for T-DNA vector sequence before alignment against the A. thaliana nuclear genome sequence obtained from MAtDB version 110103 (16) using BLASTN. A "genome hit" was defined as a FST of at least 30 bp in length with expectation values smaller than $5 \times 10^{-4}$. Resulting data were stored in the SimpleSearch database (15) that can be accessed at http: //www.mpiz-koeln.mpg.de/GABI-Kat/.

\section{RESULTS AND DISCUSSION}

We developed and tested several approaches to improve the quality of FSTs, resolve amplification of multiple fragments, and reduce consequences of plasmid-vector contamination of plant genomic DNA to establish a pipeline for efficient and economical high-throughput generation of T-DNA FSTs. The evaluation experiments were carried out on a sample of 363 lines transgenic for pAC161. Based on the pAC161 sequence, BfaI (CTAG) was selected as the restriction enzyme of choice for generation of DNA fragments spanning the T-DNA to genomic DNA junctions (Figure 1).

The common procedure for adaptor ligation-based FST production required, for each border, two nested amplifications and one cycle-sequencing reaction. When applied to both the left and right borders (four amplifica- 
tions plus two sequencing reactions), about $66 \%$ of the lines yielded at least one genome hit, with an average of 0.72 genome hits per plant (Table 2, Method I). To prevent amplification of plasmid-borne $B f a I$ fragments, we tested the inclusion of a restriction enzyme digest that splits such fragments. Two appropriate enzymes were used in the postligation digestion, XhoI for LB and PaeI (equals SphI) for RB amplifications. Both enzymes are 6-bp cutters, and the losses of FSTs containing the sites for these enzymes were estimated to be moderate. Moreover, cleavage of the plant genomic DNA with XhoI (CTCGAG) is impaired by the CG methylation found in plants, and thus, the sites are protected, in contrast to the DNA derived from the free-living agrobacteria. Inspection of the resulting data (Table 2, Method II) showed a more than $10 \%$ increase in the number of LB-originated FSTs after $\mathrm{XhoI}$ digestion. No difference was found when RB-specific amplifications were performed either with or without PaeI treatment. There is no definite explanation for the latter case, because agarose gel analyses demonstrated that plasmid-borne PCR fragments (512 bp) disappeared after PaeI digestion. It could be that gains in the number of FSTs yielded were compensated by losses resulting from the use of unprotected PaeI (GCATGC) sites.

Another approach to avoid amplification of free plasmid-borne fragments involved a special design of adaptorspecific primers for the second amplification reaction (Table 2, Method III). The primers adpA, adpC, adpG, and adpT are homologous to the $3^{\prime}$ part of the adaptor sequence and encompass a $B f a \mathrm{I}$ site and extend by the last 3' end nucleotide into the adjacent plant sequence (Table 1). The last $3^{\prime}$ end nucleotide is different in all four adaptor primers. In order to prevent amplification of free plasmid-borne fragments with T-DNA borders, only three adaptor primers were used in the PCR step for each border, and one primer containing the $3^{\prime}$ end nucleotide corresponding to the plasmid fragment was omitted (adpT for LB and adpC for RB, respectively). It was anticipated that template fragments with bases that are noncomplementary to the $3^{\prime}$ end of the primer would not be amplified. However, one-fourth of all FSTs was expected to be lost, as not only free plasmid-borne fragments were incapable of amplifying, but also those FSTs that contain the corresponding nucleotide adjacent to the $B f a \mathrm{I}$ site in the plant DNA. Indeed, with this method, we observed more losses than gains. The number of FSTs obtained for both the LB (41\%) and the RB (18\%) were significantly lower compared to the ordinary procedure with T726 adaptor primer.

The use of the $3^{\prime}$ end specific adaptor primers was also attempted to resolve multiband patterns to single-band ones. Preliminary experiments that used only one of the $3^{\prime}$ end specific adaptor primers $(\operatorname{adpA}, \operatorname{adpC}, \operatorname{adpG}$, or adpT) demonstrated that this was possible (data not shown). However, it was not considered economical (due to high sequencing costs) to perform four different sequencing reactions per border. Therefore, two second-amplification reactions were performed for each line followed by sequencing. Each of the two reactions combined two adaptor primers, either adpA and adpC or adpG and adpT.

Combining the XhoI digestion with a specific primer set resulted in $66 \%$ (adpA and adpC) and 74\% (adpG and adpT) genome hits at LB. After subtracting the $33 \%$ common hits produced by both reactions, an average 1.05 hits/line was obtained from the LB amplifications and $86 \%$ of all lines contributing an FST (Table 2, Method IV). Including RB reactions increased the yield further (Table 2, Method V). However, the RB yielded only $14 \%$ (adpA and adpC) and 20\% (adpG and adpT) FSTs with 25\% common hits (Table 2, Method V). The difference in amplification efficiency between LB and RB has been noticed before (17), but no quantitative data was provided. Part of an explanation for the difference could be that many of the $\mathrm{RB}$ sequences are present in inverted, head-to-head T-DNA arrangements (LB-RB:RB-LB) and are therefore not productive in FST generation. In fact, many of the $33 \%$ common genome hits at LB are from two LB-derived FSTs in opposite orientation.

With application of XhoI digestion only, $67 \%$ of the analyzed lines yielded FSTs from LB. For LB, the use of the 
specific primer sets increased FST yield to $86 \%$. If the contribution from RB amplifications is considered, 90\% of the lines produced at least one FST with a yield of 1.22 hits/line. These values are in agreement with genetic analyses of the GABI-Kat population (segregation for sulfadiazine resistance) where we found approximately 1.3 insertions per line. We note that not all inserted T-DNAs are detected by genetic analysis. However, in terms of productivity, performing the two additional RB amplifications was not economical. Therefore, FST generation at RB was omitted from the pipeline.

Agarose gel separation is commonly used either for purification of PCR fragments before sequencing $(18,19)$ or for fragment "picking" followed by reamplification (17). These tedious and DNA-damaging procedures greatly hamper automation of the FSTs' production process. Using additional restriction digestion and selective primers, we avoided agarose gel elecrophoresis, which considerably accelerated the pipeline. The 96 -well format was conveniently kept throughout the whole procedure.

With the assumption of a $50 \%$ GC content, every second line carrying two T-DNA insertions and/or generating a double PCR band gel pattern should be resolved by our method and produce two different FSTs. The remaining lines could still produce two overlapping sequences in one sequence file. For three or more fragments, the situation is more complicated. Some of the multiple-band patterns were resolved by computational trimming of the sequence files. In the case of two overlapping sequences, the $5^{\prime}$ part of the sequence trace was of poor quality. However, $3^{\prime}$ parts of the trace, corresponding to the longest PCR fragment, yielded unambiguous reads and produced valuable FSTs.

In summary, the final FST production pipeline included an additional restriction digestion and no gel purification. The number of amplification reactions per line was reduced from four to three (LB only), and yet the percentage of lines that yielded at least one FST was increased. We found an average of 1.05 genome hits per analyzed plant. We have currently generated more than 58,000 FSTs from GABIKat using the pipeline described. These FSTs are available to assist the scientific community with the study of gene function relationships in A. thaliana.

\section{ACKNOWLEDGMENTS}

We thank the German Plant Genomics Research Program (GABI) and the DNA core facility (ADIS) teams at Max-Planck-Institute for Plant Breeding Research (MPIZ) for generous help. Many thanks also to Heinz Saedler for continuous support, and to Seth J. Davis for critically reading the manuscript. The GABI-Kat project was supported by the Federal Ministry of Education and Research $(B M B F)$ in the context of German plant genomics program GABI (Förderkennzeichen 0312273).

\section{REFERENCES}

1.Cooley, L., R. Kelley, and A. Spradling. 1988. Insertional mutagenesis of the Drosophila genome with single P elements. Science 239:1121-1128.

2.Kumar, A., S. Vidan, and M. Snyder. 2002. Insertional mutagenesis: transposon-insertion libraries as mutagens in yeast. Methods Enzymol. 350:219-229.

3.Thorneycroft, D., S.M. Sherson, and S.M. Smith. 2001. Using gene knockouts to investigate plant metabolism. J. Exp. Bot. 52: 1593-1601.

4.Yelick, P.C. and T.F. Schilling. 2002. Molecular dissection of craniofacial development using zebrafish. Crit. Rev. Oral Biol. Med. 13: 308-322

5.McKinney, E.C., N. Ali, A. Traut, K.A. Feldmann, D.A. Belostotsky, J.M. McDowell, and R.B. Meagher. 1995. Sequence-based identification of T-DNA insertion mutations in Arabidopsis: actin mutants act2-1 and act41. Plant J. 8:613-622.

6.Krysan, P.J., J.C. Young, F. Tax, and M.R. Sussman. 1996. Identification of transferred DNA insertions within Arabidopsis genes involved in signal transduction and ion transport. Proc. Natl. Acad. Sci. USA 93: 8145-8150.

7.Clough, S.J. and A.F. Bent. 1998. Floral dip: a simplified method for Agrobacterium-mediated transformation of Arabidopsis thaliana. Plant J. 16:735-743.

8.The Arabidopsis Genome Initiative. 2000. Analysis of the genome sequence of the flowering plant Arabidopsis thaliana. Nature 408:796-815.

9. Samson, F., V. Brunaud, S. Balzergue, B. Dubreucq, L. Lepiniec, G. Pelletier, M. Caboche, and A. Lecharny. 2002. FLAGdb/ FST: a database of mapped flanking insertion sites (FSTs) of Arabidopsis thaliana T-DNA transformants. Nucleic Acids Res. 30:94-97.

10.Sessions, A., E. Burke, G. Presting, G. Aux, J. McElver, D. Patton, B. Dietrich, P. Ho, et al. 2002. A high-throughput Arabidopsis reverse genetics system. Plant Cell 14:29852994

11.Alonso, J.M., A.N. Stepanova, T.J. Leisse, C.J. Kim, H. Chen, P. Shinn, D.K. Stevenson, J. Zimmerman, et al. 2003. Genomewide insertional mutagenesis of Arabidopsis thaliana. Science 301:653-657.

12.Castle, L.A., D. Errampalli, T.L. Atherton, L.H. Franzmann, E.S. Yoon, and D.W. Meinke. 1993. Genetic and molecular characterization of embryonic mutants identified following seed transformation in Arabidopsis. Mol. Gen. Genet. 241:504-514.

13.Doyle, J.J. and J.L. Doyle. 1987. A rapid DNA isolation procedure for small quantities of fresh leaf tissue. Phytochem. Bull. 19: 11-15.

14.Steiner-Lange, S., M. Gremse, M. Kuckenberg, E. Nissing, D. Schachtele, N. Spenrath, M. Wolff, H. Saedler, et al. 2001. Efficient identification of Arabidopsis knockout mutants using DNA-arrays of transposon flanking sequences. Plant Biol. 3:391-397.

15.Li, Y., M.G. Rosso, N. Strizhov, P. Viehoever, and B. Weisshaar. 2003. GABI-Kat SimpleSearch: a flanking sequence tag (FST) database for the identification of T-DNA insertion mutants in Arabidopsis thaliana. Bioinformatics 19:1441-1442.

16.Schoof, H., P. Zaccaria, H. Gundlach, K. Lemcke, S. Rudd, G. Kolesov, R. Arnold, H.W. Mewes, et al. 2002. MIPS Arabidopsis thaliana Database (MAtDB): an integrated biological knowledge resource based on the first complete plant genome. Nucleic Acids Res. 30:91-93.

17.Balzergue, S., B. Dubreucq, S. Chauvin, I. Le-Clainche, F. Le Boulaire, R. de Rose, F. Samson, V. Biaudet, et al. 2001. Improved PCR-walking for large-scale isolation of plant T-DNA borders. BioTechniques 30:496-504.

18.Parinov, S., M. Sevugan, D. Ye, W.C. Yang, M. Kumaran, and V. Sundaresan. 1999. Analysis of flanking sequences from dissociation insertion lines: a database for reverse genetics in Arabidopsis. Plant Cell 11: 2263-2270.

19.Szabados, L., I. Kovacs, A. Oberschall, E. Abraham, I. Kerekes, L. Zsigmond, R. Nagy, M. Alvarado, et al. 2002. Distribution of 1000 sequenced T-DNA tags in the Arabidopsis genome. Plant J. 32:233-242.

Received 4 August 2003; accepted 10 September 2003.

Address correspondence to Bernd Weisshaar, Bielefeld University, Department of Biology, D-33594 Bielefeld, Germany. email: genomforschung@uni-bielefeld.de 